\title{
Construction of Global Acyl Lipid Metabolic Map by Comparative Genomics and Subcellular Localization Analysis in the Red Alga Cyanidioschyzon merolae
}

\author{
Natsumi Mori ${ }^{1,2}$, Takashi Moriyama ${ }^{1,2}$, Masakazu Toyoshima ${ }^{1,2}$ and Naoki Sato 1,2* \\ ${ }^{1}$ Department of Life Sciences, Graduate School of Arts and Sciences, University of Tokyo, Tokyo, Japan, ${ }^{2}$ Japan Science \\ and Technology Agency, Core Research for Evolutional Science and Technology, Tokyo, Japan
}

\section{OPEN ACCESS}

Edited by:

Toshiya Muranaka,

Osaka University, Japan

Reviewed by:

Qing Liu,

Commonwealth Scientific and Industrial Research Organisation,

Australia

Akira Oikawa,

Yamagata University, Japan

${ }^{*}$ Correspondence: Naoki Sato

naokisat@bio.c.u-tokyo.ac.jp

Specialty section:

This article was submitted to Plant Metabolism and Chemodiversity,

a section of the journal

Frontiers in Plant Science

Received: 26 April 2016

Accepted: 15 June 2016

Published: 30 June 2016

Citation:

Mori N, Moriyama T, Toyoshima M and Sato N (2016) Construction of Global Acyl Lipid Metabolic Map by

Comparative Genomics and

Subcellular Localization Analysis in the Red Alga Cyanidioschyzon merolae.

Front. Plant Sci. 7:958.

do: 10.3389/fpls.2016.00958
Pathways of lipid metabolism have been established in land plants, such as Arabidopsis thaliana, but the information on exact pathways is still under study in microalgae. In contrast with Chlamydomonas reinhardtii, which is currently studied extensively, the pathway information in red algae is still in the state in which enzymes and pathways are estimated by analogy with the knowledge in plants. Here we attempt to construct the entire acyl lipid metabolic pathways in a model red alga, Cyanidioschyzon merolae, as an initial basis for future genetic and biochemical studies, by exploiting comparative genomics and localization analysis. First, the data of whole genome clustering by Gclust were used to identify 121 acyl lipid-related enzymes. Then, the localization of 113 of these enzymes was analyzed by GFP-based techniques. We found that most of the predictions on the subcellular localization by existing tools gave erroneous results, probably because these tools had been tuned for plants or green algae. The experimental data in the present study as well as the data reported before in our laboratory will constitute a good training set for tuning these tools. The lipid metabolic map thus constructed show that the lipid metabolic pathways in the red alga are essentially similar to those in A. thaliana, except that the number of enzymes catalyzing individual reactions is quite limited. The absence of fatty acid desaturation to produce oleic and linoleic acids within the plastid, however, highlights the central importance of desaturation and acyl editing in the endoplasmic reticulum, for the synthesis of plastid lipids as well as other cellular lipids. Additionally, some notable characteristics of lipid metabolism in C. merolae were found. For example, phosphatidylcholine is synthesized by the methylation of phosphatidylethanolamine as in yeasts. It is possible that a single 3-ketoacyl-acyl carrier protein synthase is involved in the condensation reactions of fatty acid synthesis in the plastid. We will also discuss on the redundant $\beta$-oxidation enzymes, which are characteristic to red algae.

Keywords: comparative genomics, subcellular localization, lipid metabolism, red alga, Cyanidioschyzon merolae 


\section{INTRODUCTION}

Oil production by microalgae is a recent trend, but very little is known about the lipid metabolic pathways in microalgae, except in the green alga Chlamydomonas reinhardtii, in which considerable knowledge is rapidly accumulating (Li-Beisson et al., 2015). The researchers on microalgae still use obscure views on the lipid metabolic pathways, which are mostly the products of analogy with the pathways obtained in better-studied organisms, such as Arabidopsis thaliana and C. reinhardtii. The overview on the lipid metabolism in plants (mostly Arabidopsis) is summarized below as a reference (Supplementary Figure 1).

Plastids are the major site of fatty acid synthesis. 16or 18-carbon fatty acids, such as palmitic, stearic and oleic acids, are synthesized in the plastids (Joyard et al., 2010). Mitochondrial fatty acid synthesis is also known as a minor pathway (Wada et al., 1997; Gueguen et al., 2000). Verylong-chain fatty acids (more than 20-carbon fatty acids) are synthesized in the endoplasmic reticulum (ER) by elongation of 16- or 18-carbon acyl-coenzyme A (CoA) produced in the plastids (Haslam and Kunst, 2013). Four major plastid membrane lipids, monogalactosyldiacylglycerol (MGDG), digalactosyldiacylglycerol (DGDG), sulfoquinovosyldiacylglycerol (SQDG) and phosphatidylglycerol (PG), are synthesized within the plastids (Joyard et al., 2010). On the other hand, the ER is the major site of synthesis of phospholipids, namely, phosphatidylcholine (PC), phosphatidylethanolamine (PE), phosphatidylserine (PS), phosphatidylinositol (PI) and PG, as well as the site of triacylglycerol (TAG) synthesis in plants (Li-Beisson et al., 2013). The glycolipids and phospholipids are further modified by formation of double bonds within their acyl chains, catalyzed by various acyl lipid desaturases in the plastids and the ER. Once synthesized, TAG is accumulated in the organelle called lipid body and is used as energy and carbon sources during germination (Chapman et al., 2012). Peroxisomes are the major site of $\beta$-oxidation pathway in plants (Graham and Eastmond, 2002; Poirier et al., 2006).

We are interested in red algae as new resources for future bioengineering. Red algae have attracted less attention in biochemical and genomic researches, mainly because many of them are marine macrophytes (Nakamura et al., 2013). In contrast, Cyanidioschyzon merolae is a unicellular red alga, which is easy to grow and to manipulate in the laboratory. It was originally isolated from an acidic hot spring, and grows by binary fission. Its habitat is warm (up to $50^{\circ} \mathrm{C}$ ) and acidic ( $\mathrm{pH} 1.5-$ 2.5) land water containing sulfuric acid. This alga is a versatile model alga, because its complete sequences of the nuclear, plastid and mitochondrial genomes have been determined (Matsuzaki et al., 2004; Nozaki et al., 2007). The small number of protein coding genes (4775 nuclear genes) in C. merolae as well as the experimental techniques of transformation (Ohnuma et al., 2008, 2014) made it an ideal model alga for reverse-genetic analysis. In addition, its simple cellular organization (a single plastid, a single mitochondrion, a single peroxisome) makes it a suitable organism for the study of cytological analysis, such as subcellular localization of proteins.
We already published two lines of studies on $C$. merolae. In one of them lipid metabolism was analyzed by radiolabeling and a preliminary genomic analysis (Sato and Moriyama, 2007), showing various points of uniqueness in lipid metabolism has been noted. First, this alga does not possess stearoyl-acyl-carrierprotein $(\mathrm{ACP})$ desaturase, which is universally present in green plants and algae. This makes ER, rather than the plastid, the major site of desaturation of fatty acids and lipids. The "coupled pathway" was proposed for the synthesis of galactolipids in the plastid. Second, C. merolae lacks polyunsaturated fatty acids containing three or more double bonds, such as linolenic acid and arachidonic acid. Third, this alga has MgdA and DgdA homologs involved in the galactolipid synthesis in cyanobacteria, as well as a eukaryotic type MGD1 (for a review, see Sato and Awai, 2016). A recently published work in this line (Toyoshima et al., 2016) analyzed TAG accumulation in the nitrogen deficiency. Sato et al. (2016b) studied biosynthesis of PC by labeling with radioactive phosphate.

In another line of study (Moriyama et al., 2014a), we used comparative genomics to identify all 75 enzymes involved in the central carbohydrate metabolism in C. merolae, which were then subjected to global, experimental analysis of subcellular localization. Although, most of these enzymes are soluble proteins, we noticed some errors in prediction of plastid or mitochondrial localization by the available prediction tools. By extending the previous study (Sato and Moriyama, 2007; Sato et al., 2016b), we now attempt to identify all lipid metabolic enzymes in $C$. merolae by comparative genomics, and to analyze experimentally the subcellular localization of all these enzymes. This is a new challenge, because a large part of these enzymes are insoluble, membrane proteins. In fact, preliminary prediction results suggested a strange compartmentation of lipid metabolic enzymes, very different from that in plants and algae. Subcellular localization of enzymes has been predicted by the computer programs, such as TargetP (Emanuelsson et al., 2000) and WoLF PSORT (Horton et al., 2007), based on neural network algorithm that gives good estimates after proper training. In this respect, the existing programs might be suitable for analysis of proteins of land plants, but not for algal proteins. Recently, the PredAlgo was made as a prediction tool of green algal proteins using datasets of proteome analysis in C. reinhardtii (Tardif et al., 2012). This is still not suitable for red algal proteins, as we will see later.

In the present study, we first report on the construction of a list (or database) of all enzymes involved in acyl lipid metabolism in C. merolae by exploiting the whole genome clustering by the Gclust software (Sato, 2009) with various photosynthetic and non-photosynthetic organisms. We identified the lipid metabolic enzymes in algae and cyanobacteria by using the orthologs in A. thaliana as queries. Second, we determined the subcellular localization of these enzymes by the GFP- or hemagglutinin (HA) tag-fusion techniques. Third, we created probable pathway maps of lipid metabolism based on these results. We confirmed that the major sites of lipid metabolism are plastid and ER in $C$. merolae as in plants, although the pathways in $C$. merolae consist of a minimal set of enzymes. An exception is the $\beta$-oxidation enzymes, of which we found two sets. 


\section{MATERIALS AND METHODS}

\section{Construction of Comparative Database of Acyl Lipid Metabolic Enzymes in Algae and Cyanobacteria}

To build the database of lipid metabolic enzymes in algae and cyanobacteria, we searched for enzymes related to synthesis of fatty acids and lipids, lipid degradation, $\beta$-oxidation and lipid trafficking in C. merolae 10D, C. reinhardtii CC-503, Synechocystis sp. PCC 6803 and Anabaena sp. PCC 7120 using the Gclust database (Sato, 2009; http://gclust.c.u-tokyo.ac.jp/) based on the genomic data of $A$. thaliana. We used the TAIR database (http://www.arabidopsis.org/), the KEGG database (http://www. genome.jp/kegg/), C. reinhardtii v5.5 in the Phytozome database (http://phytozome.jgi.doe.gov) and the Cyanidioschyzon merolae Genome Project (http://merolae.biol.s.u-tokyo.ac.jp/) to obtain the genomic data of these organisms.

\section{Growth of Organism}

C. merolae cells were cultured in $100 \mathrm{ml}$ of $2 \times$ Allen's medium (pH 2.5; $20 \mathrm{mM}\left(\mathrm{NH}_{4}\right)_{2} \mathrm{SO}_{4}, 4 \mathrm{mM} \mathrm{KH_{2 }} \mathrm{PO}_{4}, 2 \mathrm{mM} \mathrm{MgSO}_{4}$, $1 \mathrm{mM} \mathrm{CaCl}, 50 \mu \mathrm{M} \mathrm{FeCl}_{3}, 10 \mu \mathrm{M}$ ethylenediaminetetraacetic acid, $200 \mathrm{nM} \mathrm{CuSO}_{4}, 200 \mathrm{nM} \mathrm{ZnSO}_{4}, 160 \mathrm{nM} \mathrm{Na}_{2} \mathrm{MoO}_{4}, 300 \mathrm{nM}$ $\mathrm{CoCl}_{2}, 16 \mu \mathrm{M} \mathrm{H}_{3} \mathrm{BO}_{3}, 3.2 \mu \mathrm{M} \mathrm{MnCl}_{2}$ ) using shaking flasks under continuous red light $\left(30 \mu \mathrm{mol} \mathrm{m}^{-2} \mathrm{~s}^{-1}\right)$ at $40^{\circ} \mathrm{C}$.

\section{Plasmid Construction}

pCG1 vector contains APCC promoter of C. merolae, sGFP (S65T) gene and NOS terminator (Watanabe et al., 2011). First, we replaced $s G F P$ (S65T) gene with EGFP gene in pCG1 vector and named it pCEG1 vector. To obtain pCG1 vector without sGFP (S65T) gene, we performed PCR using pCG1 vector as a template and a primer set (5'-AGCGGCCGCCCGGCTGCA GATCGTTCAAACATTTG- $3^{\prime}$ and $5^{\prime}$-GGATCCTCTAGAGGT CAACGAACGAAGAAACACAG-3'). EGFP gene was amplified using pEGFP vector (Clontech Laboratories, Mountain View, CA, USA) as a template and a primer set ( $5^{\prime}$-ACCTCTAGA GGATCCatggtgagcaagggcgagga- $3^{\prime}$ and $5^{\prime}$-AGCCGGGCGGCC GCTttacttgtacagctcgtcca-3'; The sequences in uppercase letters indicate common sequences of pCEG1 vector required for the cloning using the In-Fusion Cloning Kit; Clontech Laboratories.) and, inserted downstream of APCC promoter in PCG1 vector without $s G F P$ (S65T) gene using the In-Fusion Cloning Kit.

We prepared a sequence alignment for each lipid metabolic enzyme by Clustal X 2.0.10 (Larkin et al., 2007) and determined the region of $N$-terminal extended sequence, which is a putative transit peptide in C. merolae. A DNA fragment of $\mathrm{N}$-terminal extended sequence was amplified using the $C$. merolae genome, and inserted into pCEG1 vector restricted with $\mathrm{XbaI}$ using the In-Fusion Cloning Kit. For small proteins, such as ACBP or mtACP, the GFP-fused construct was prepared using the fulllength gene as an insert. For constructs of HA-tagged proteins, full length DNA fragments were inserted into $\mathrm{pBSHAb}-\mathrm{T}^{\prime}$ vector (Ohnuma et al., 2008) containing APCC promoter (Moriyama et al., 2014a) restricted with PacI using the In-Fusion Cloning Kit. For constructs of EGFP-fused $C$-terminal peptide of $\beta$-oxidation enzymes, first we performed PCR using pCEG1 vector as a template and a primer set (5'-TAAAGCGGCCGCCCGGCTG CAGATCGTTCA- $3^{\prime}$ and $5^{\prime}$-CTTGTACAGCTCGTCCATGCCG AGAGTGAT- $3^{\prime}$ ). A DNA fragment of $C$-terminal peptide of $\beta$ oxidation enzymes was amplified using the $C$. merolae genome. These PCR products were cloned with the In-Fusion Cloning Kit. Supplementary Table 1 is a list of primers used for amplification of inserts.

\section{Transformation and Immunostaining}

Transformation of $C$. merolae cells was performed using the polyethylene glycol-method as described earlier (Moriyama et al., 2014b). If necessary, transformed cells expressing a GFPfused protein were immunostained according to Moriyama et al. (2014a). C. merolae cells bearing HA-tagged proteins were immunostained with the Tyramide Staining Amplification (TSA) Kit (Invitrogen) according to Moriyama et al. (2014a). Transformed or immunostained cells were observed with a fluorescence microscope (Moriyama et al., 2014a). Genetic manipulation experiments were carried out according to the guideline of the Genetic Recombination Experiment Safety Control Committee in Tokyo Univerity.

\section{RESULTS}

\section{Search for Lipid Metabolic Enzymes in C. merolae}

We searched for enzymes related to acyl lipid metabolism in $C$. merolae, C. reinhardtii, Synechocystis sp. PCC 6803 and Anabaena sp. PCC 7120 using the Gclust database (Sato, 2009) using the 373 known enzymes involved in the acyl lipid metabolism in A. thaliana as queries (Supplementary Table 2). The Gclust software is a tool of whole genome clustering, which is suited for identifying highly related orthologs (conserving their entire domain structure) over prokaryotes and eukaryotes, allowing the presence or absence of a transit peptide. Proteins sharing a single domain might not be identified in the same cluster, but can be picked up as a related group. As a result, 121, 163, 51, and 59 putative enzymes involved in the acyl lipid metabolism were detected in C. merolae, C. reinhardtii, Synechocystis sp. PCC 6803 and Anabaena sp. PCC 7120, respectively. Table $\mathbf{1}$ is a summary of enzymes involved in the synthesis of fatty acids and lipids as well as $\beta$-oxidation in $C$. merolae. Other enzymes, such as lipases and lipid transporters in the plastid, are summarized in Supplementary Table 3.

We estimated subcellular localization of these enzymes using three prediction tools for subcellular localization, namely TargetP (Emanuelsson et al., 2000), WoLF PSORT (Horton et al., 2007) and PredAlgo (Tardif et al., 2012; Table 1, Supplementary Table 3). TargetP and WoLF PSORT are predictors for subcellular localization of eukaryotic proteins including plants. PredAlgo is a prediction tool for subcellular localization of green algal proteins. A number of differences were detected in the localization of $C$. merolae enzymes from that of the plant homologs. To examine if the localization of these enzymes are really different in plants and C. merolae, we analyzed subcellular localization of 113 putative lipid metabolic enzymes in C. merolae using the GFP- or HA tagfusion techniques. Eight enzymes encoded in the plastid genome 
TABLE 1 | List of acyl lipid metabolic enzymes in C. merolae with emphasis on the subcellular localization.

\begin{tabular}{|c|c|c|c|c|c|c|}
\hline \multirow[t]{2}{*}{ 1. Enzyme name } & \multirow{2}{*}{$\begin{array}{l}\text { 2. Abbreviation of } \\
\text { enzyme name and/or } \\
\text { gene name }\end{array}$} & \multirow[t]{2}{*}{ 3. Locus tag } & \multirow{2}{*}{$\begin{array}{l}\text { 4. Subcellular } \\
\text { localization }\end{array}$} & \multicolumn{3}{|c|}{ 5. Result of prediction of subcellular localization } \\
\hline & & & & TargetP & WoLF PSORT & PredAlgo \\
\hline \multicolumn{7}{|c|}{ FATTY ACID SYNTHESIS AND ELONGATION } \\
\hline $\begin{array}{l}\text { Acetyl-CoA carboxylase } \\
\text { (multifunctional type) }\end{array}$ & ACCase (ACC1) & CMM188C & Cyt & Other & Nuc, Cyt & Other \\
\hline \multirow{4}{*}{$\begin{array}{l}\text { Acetyl-CoA carboxylase } \\
\text { (multisubunit type) }\end{array}$} & ACCase (AccA) & CMV056C & Pt-genome & & & \\
\hline & $(\mathrm{AccB})$ & CMV134C & Pt-genome & & & \\
\hline & $(\mathrm{AccC})$ & CMS299C & $\mathrm{Pt}$ & Pt & Pt, Cyt & Mt \\
\hline & (AccD) & CMV207C & Pt-genome & & & \\
\hline $\begin{array}{l}\text { Malonyl-CoA:ACP } \\
\text { malonyltransferase }\end{array}$ & MCMT & CMT420C & $\mathrm{Pt}$ & Other & $\mathrm{Pt}$ & $\mathrm{Pt}$ \\
\hline Acetyl-CoA:ACP acetyltransferase & ACAT & Not detected & & & & \\
\hline \multirow[t]{2}{*}{ Acyl carrier protein } & $\mathrm{ACP}(\mathrm{AcpP})$ & CMV132C & Pt-genome & & & \\
\hline & $(\mathrm{mtACP})$ & CMS372C & Mt & Mt & $\mathrm{Pt}$ & Mt \\
\hline \multirow[t]{2}{*}{ 3-Ketoacyl-ACP synthase } & KAS (KAS I) & CMM286C & $\mathrm{Pt}$ & $\mathrm{Pt}$ & Mt & Other \\
\hline & (mtKAS) & CML329C & Mt & Other & $\mathrm{Pt}$ & Other \\
\hline 3-Ketoacyl-ACP reductase & KAR & CMS393C & $\mathrm{Pt}$ & $\mathrm{Pt}$ & $\mathrm{Pt}$ & Other \\
\hline 3-Hydroxyacyl-ACP dehydratase & HAD & CMI240C & $\mathrm{Pt}$ & Mt & $\mathrm{Pt}$ & Other \\
\hline Enoyl-ACP reductase & EAR & CMT381C & $\mathrm{Pt}$ & Mt & $\mathrm{Pt}$ & $\mathrm{Pt}$ \\
\hline Acyl-ACP thioesterase & AAT & $\mathrm{CMH} 111 \mathrm{C}$ & $\mathrm{Pt}$ & $\mathrm{Pt}$ & Cyt & Other \\
\hline 3-Ketoacyl-CoA synthase (Elongase) & KCS & CMD118C & ER & Pt & PM & Mt \\
\hline 3-Ketoacyl-CoA reductase & KCR & CMK172C & ER & Other & $\mathrm{Pt}$ & $\mathrm{SP}$ \\
\hline 3-Hydroxyacyl-CoA dehydratase & HCD & CMR006C & ER & $\mathrm{SP}$ & PM & Other \\
\hline Enoyl-CoA reductase & ECR & CMD146C & ER & Mt & $\mathrm{Pt}$ & Mt \\
\hline \multirow{3}{*}{$\begin{array}{l}\text { Homologs of yeast ELO genes } \\
\text { (3-Ketoacyl-CoA synthase?) }\end{array}$} & ELO-like & CMT175C & ER & Mt & $\mathrm{Pt}$ & Other \\
\hline & & CMM126C & ER & Other & Cyt & Other \\
\hline & & CML178C & Not detected & Mt & $\mathrm{Pt}$ & $\mathrm{Pt}$ \\
\hline \multicolumn{7}{|l|}{ DESATURASE } \\
\hline Stearoyl-CoA desaturase & SCD & CMM045C [1-2] & ER & Other & Cyt & Other \\
\hline Acyl lipid $\Delta 9$ desaturase & $\Delta 9 \mathrm{Des}$ & CMJ201C [1] & ER & Other & PM & $\mathrm{Pt}$ \\
\hline Acyl lipid $\Delta 12$ desaturase & $\Delta 12 \mathrm{Des}$ & CMK291C [1] & ER & Other & Pt, Pt_Mt & Other \\
\hline $\begin{array}{l}\text { Phosphatidylglycerol specific } \\
\Delta 3 \text {-trans desaturase }\end{array}$ & FAD4 & CMF187C & Not detected & Other & Nuc & Other \\
\hline \multicolumn{7}{|c|}{ SYNTHESIS OF GLYCOLIPIDS AND PG } \\
\hline $\begin{array}{l}\text { Glycerol-3-phosphate } \\
\text { acyltransferase }\end{array}$ & GPAT & CMJ027C & $\mathrm{Pt}$ & Mt & $\mathrm{Pt}$ & $\mathrm{Pt}$ \\
\hline $\begin{array}{l}\text { Lysophosphatidic acid } \\
\text { acyltransferase }\end{array}$ & LPAT & CMF185C & $\mathrm{Pt}$ & Mt & PM & Mt \\
\hline Phosphatidic acid phosphatase & PAP & Not detected & & & & \\
\hline $\begin{array}{l}\text { Monogalactosyldiacylglycerol } \\
\text { synthase }\end{array}$ & MGD1 & CMI271C & $\mathrm{Pt}$ & $\mathrm{Pt}$ & $\mathrm{Pt}$ & $\mathrm{Pt}$ \\
\hline Monoglucosyldiacylglycerol synthase & MgdA & CMT267C & ER & Mt & PM & $\mathrm{SP}$ \\
\hline Glucolipid epimerase & MgdE & Not detected & & & & \\
\hline Digalactosyldiacylglycerol synthase & $\operatorname{DgdA}$ & CMV121C [3] & Pt-genome & & & \\
\hline $\begin{array}{l}\text { Galactolipid:galactolipid } \\
\text { galactosyltransferase }\end{array}$ & SFR2 & Not detected & & & & \\
\hline UDP-sulfoquinovose synthase & SQD1 & CMR012C [5] & $\mathrm{Pt}$ & Pt & $\mathrm{Pt}$ & $\mathrm{Pt}$ \\
\hline $\begin{array}{l}\text { Sulfoquinovosyldiacylglycerol } \\
\text { synthase }\end{array}$ & SQD2 & CMR015C [5] & $\mathrm{Pt}$ & Pt & $\mathrm{Pt}$ & Mt \\
\hline
\end{tabular}


TABLE 1 | Continued

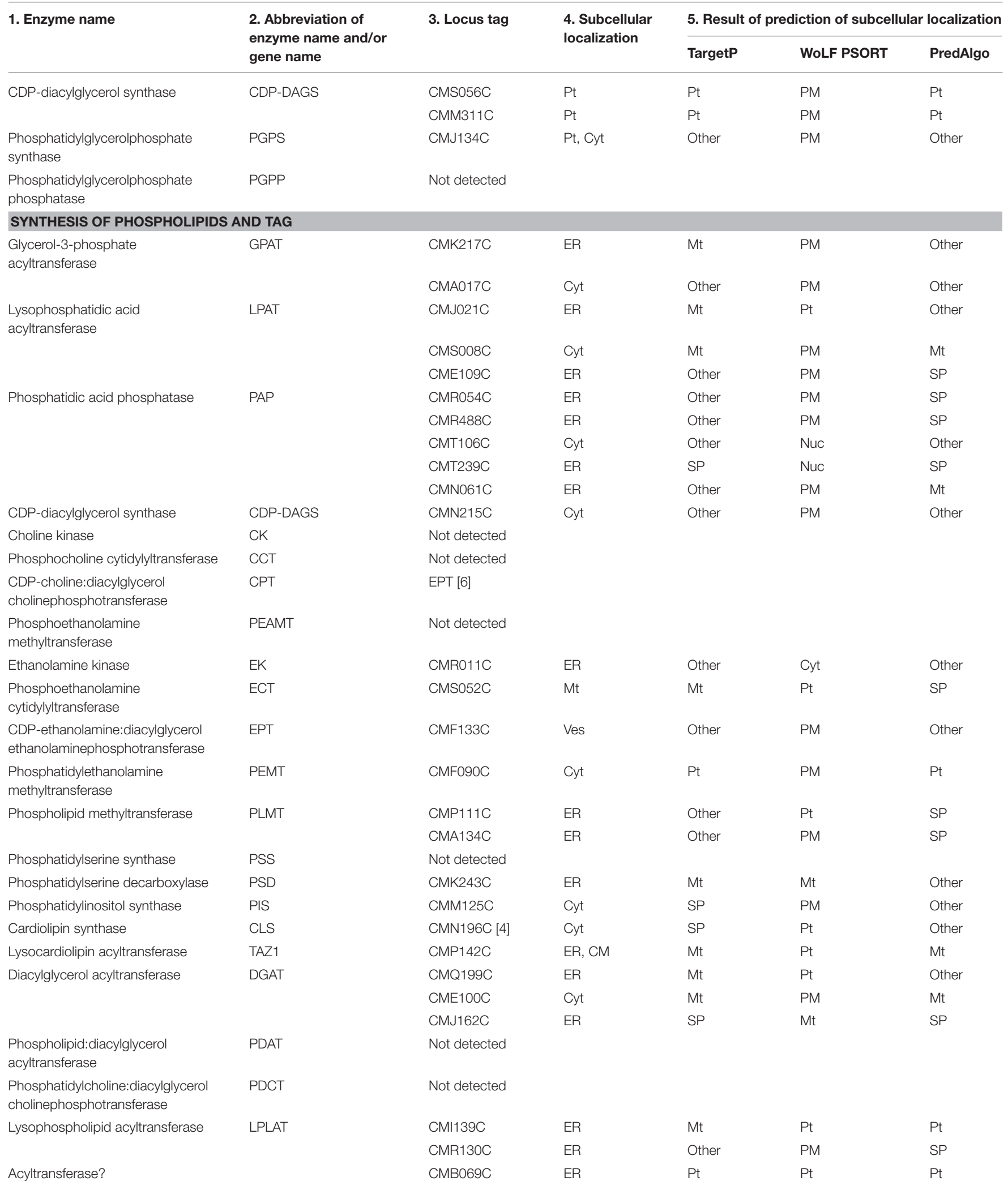


TABLE 1 | Continued

\begin{tabular}{|c|c|c|c|c|c|c|}
\hline 1. Enzyme name & $\begin{array}{l}\text { 2. Abbreviation of } \\
\text { enzyme name and/or } \\
\text { gene name }\end{array}$ & 3. Locus tag & $\begin{array}{l}\text { 4. Subcellular } \\
\text { localization }\end{array}$ & \multicolumn{3}{|c|}{ 5. Result of prediction of subcellular localization } \\
\hline Acyl-CoA oxidase & $A C X$ & CMK115C & Cyt/Cyt & Other & Cyt & Pt \\
\hline Acyl-CoA dehydrogenase & $\mathrm{ACDH}$ & CML080C & Mt & Mt & Mt & Other \\
\hline Isovaleryl-CoA dehydrogenase & IVD & СМТ072C & Mt & Mt & Nuc & Other \\
\hline Enoyl-CoA hydratase & & СМТ074C & Pt & Mt & Pt & Other \\
\hline 3-Hydroxyacyl-CoA dehydrogenase & $\mathrm{HACDH}$ & CMC137C & Mt, Cyt & Other & Pt & Mt \\
\hline Multifunctional protein & MFP & CMR380C & Ves/Cyt & Other & ER, ER_PM & SP \\
\hline \multirow[t]{2}{*}{ 3-Ketoacyl-CoA thioesterase } & KAT & CMA042C & Cyt/Nes & Other & Cyt & Mt \\
\hline & & CME087C & Mt & Mt & Pt & Mt \\
\hline
\end{tabular}

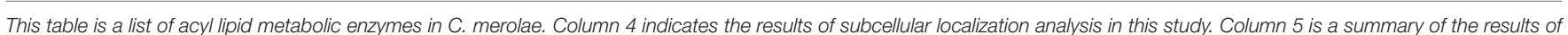

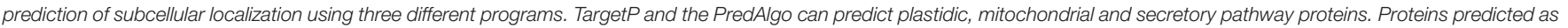

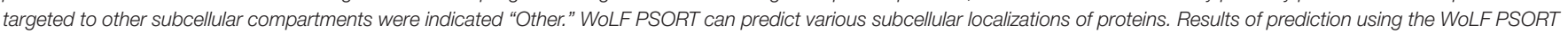

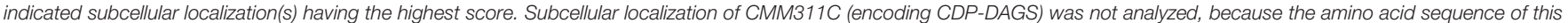

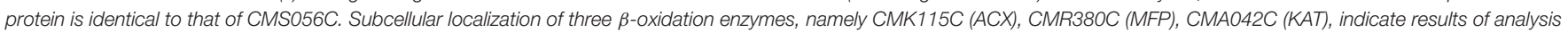

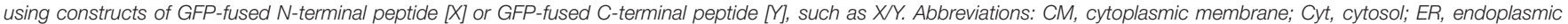

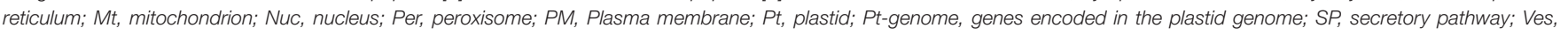

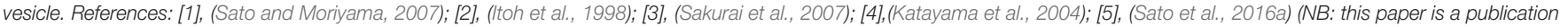
from a different group from ours); [6] (Sato et al., 2016b)

were not analyzed, because they are considered to remain in the plastid and function therein.

\section{Global Analysis of Subcellular Localization of Lipid Metabolic Enzymes in C. merolae}

Plastid, mitochondrion, nucleus and peroxisome are easily observed without overlapping in the C. merolae cell. Additionally, ER is located at the periphery of the nucleus (Yagisawa et al., 2012; Figure 1A). To determine the subcellular localization of lipid metabolic enzymes, each of the $N$-terminal transit peptide region of these enzymes was fused at the $N$-terminus of GFP (Figure 1B), or three repeats of the HA tag were fused at the $C$ terminus of full length of these enzymes (Figure 1C). The GFPor HA tag-fused proteins were expressed under the control of APCC promoter of C. merolae (Watanabe et al., 2011). When GFP fluorescence was not detected clearly, transformed cells were fixed and immunostained with anti-GFP antibody. HAtagged proteins were detected by immunostaining with anti-HA antibody. Figure 1D shows examples of subcellular localization of GFP-fusion proteins that were localized to the plastid (malonyl-CoA:ACP malonyltransferase; MCMT; CMT240C), the mitochondrion (phosphoethanolamine cytidylyltransferase; ECT; CMS052C), the cytosol (multifunctional type acetyl-CoA carboxylase; ACC1; CMM188C), the ER (phosphatidic acid phosphatase; PAP; CMT239C and diacylglycerol acyltransferase; DGAT; CMQ199C), the peroxisome (catalase; CMI050C; Imoto et al., 2013), and dually localized to the ER and the cytoplasmic membrane (flippase; ALA1; CMR306C). GFP-fused CMT239C was targeted to the periphery of the nucleus. In GFP-fused CMQ199C, immunofluorescence was observed as a fog in the cytosol. We supposed that both GFP-fusion proteins that were not homogeneously distributed in the cytosol were localized in the ER. In this way, the localization is unambiguously identified, because each organelle is present as a single entity. There is no complication due to crowding of organelles, which is usually the case in plant cells. Micrographs of 3-ketoacylCoA synthase (KCS; CMD118C, Supplementary Figure 2C), phospholipase A1 (PLA1; CMH204C, Supplementary Figure 5A), and phosphatidylinositol-4-phosphate 5-kinase (PIP5K; CME153C, Supplementary Figure 6B) were showed as examples of immunostained for ER, cytosol and both cytosol and cytoplasmic membrane in C. merolae cells. Other fluorescence micrographs are shown in Supplementary Figures 2-6. Results of subcellular localization analysis of lipid metabolic enzymes are summarized in Table 1, Supplementary Table 3.

\section{Fatty Acid Synthesis and Elongation}

In $C$. merolae, 14 proteins related to fatty acid synthesis were detected by the Gclust analysis (Table 1). Most of these enzymes, namely MCMT (CMT420C), 3-ketoacyl-ACP reductase (KAR; CMS393C), 3-hydroxyacyl-ACP dehydratase (HAD; CMI240C), enoyl-ACP reductase (EAR; CMT381C) and acyl-ACP thioesterase (AAT; CMH111C), were localized in the plastid (Figure 1D, Supplementary Figure 2A). Plastidic ACP is encoded in the plastid genome, and was not analyzed. In addition, C. merolae has two types of acetyl-CoA carboxylases (ACCases), namely multifunctional type and multisubunit type, as in plants. Multifunctional type ACCase (ACC1; CMM188C) was localized in the cytosol (Figure 1D). A product of nuclear AccC gene (CMS299C) encoding the subunit C of multisubunit type ACCase was targeted to the plastid (Supplementary Figure 2A). Other subunits of multisubunit type ACCase (subunits A, B, and D) are encoded in the plastid genome, so the multisubunit type ACCase is identified as a plastid enzyme. CMM286C 


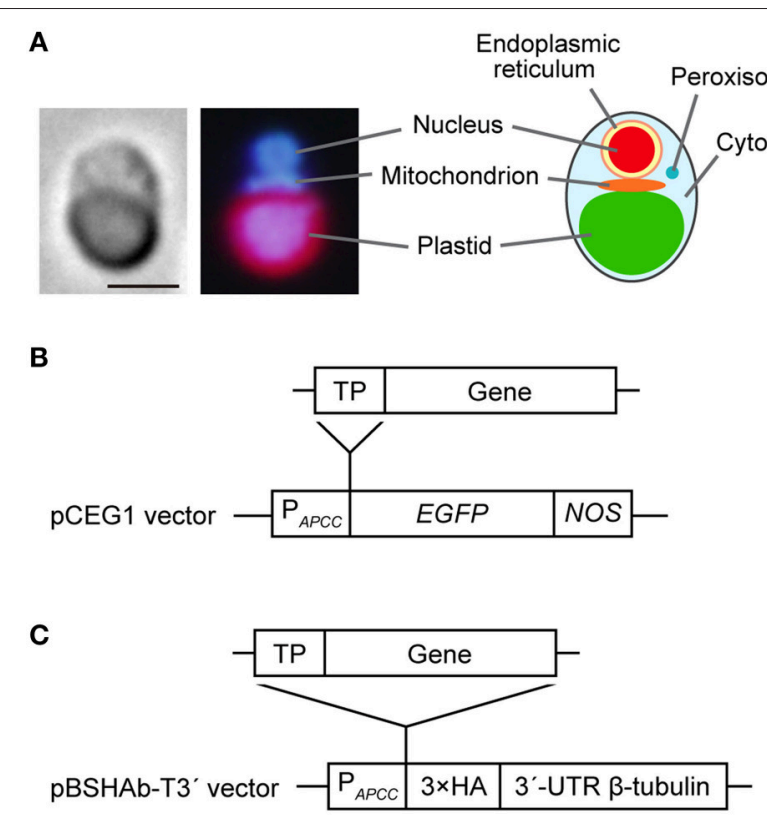

D

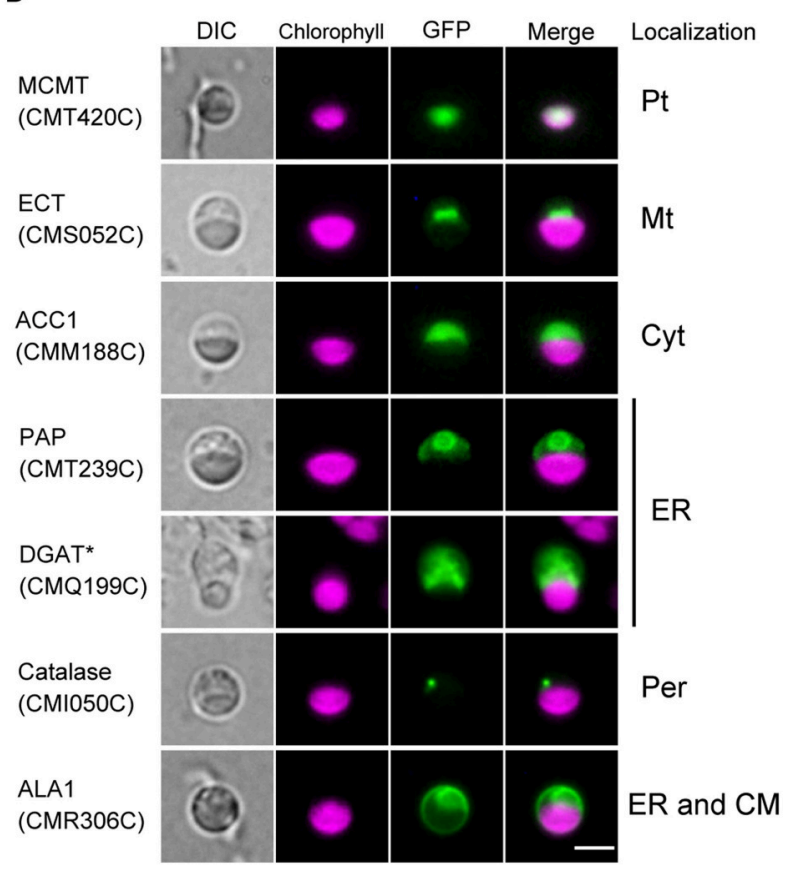

FIGURE 1 | Examples of subcellular localization of GFP-fused proteins. (A) Fluorescence microscopic images showing a C. merolae cell stained with 4',6-diamidino-2-phenylindole (DAPI; left). The illustration is a summary of cellular structure of $C$. merolae cell (right). Bar $=2 \mu \mathrm{m}$. (B) Structure of a construct expressing a GFP-fused protein. pCEG1 vector contains APCC promoter of $C$. merolae $\left(P_{A P C C}\right)$ (Watanabe et al., 2011), gene for enhanced green fluorescence protein (EGFP) and NOS terminator (NOS). Genomic fragment of $N$-terminal transit peptide (TP) of lipid metabolic enzymes was inserted into PCEG1 vector and cloned. This construct was transformed into C. merolae cells for transient expression. If necessary, transformed cells were immunostained with anti-GFP antibody. (C) Structure of a construct expressing an HA-tagged protein. pBSHAb-T3' vector (Ohnuma et al., 2008)

(Continued)

\section{FIGURE 1 | Continued}

contains three repeats of $\mathrm{HA}$ tag $(3 \times \mathrm{HA})$ and $3^{\prime}$-untranslated region (UTR) of $\beta$-tubulin of $C$. merolae. A full-length sequence of candidate genes was

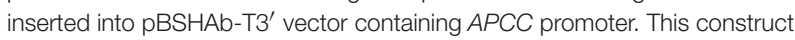
was transformed into $C$. merolae cells, and then subcellular localization of HA-tagged proteins was detected by immunostaining using anti-HA antibody. (D) Fluorescence micrographs of $C$. merolae cells transiently expressing GFP-fused proteins. These images show that subcellular localization of GFP-fused proteins to plastid (Pt), mitochondrion (Mt), cytosol (Cyt), ER, peroxisome (Per), and dual localization of ER and cytoplasmic membrane (CM). GFP-fused PAP was localized in the periphery of the nucleus. In GFP-fused DGAT, GFP fluorescence was observed as a fog in cytosol. We, therefore, supposed these proteins as ER-localized enzymes. An asterisk indicates an enzyme, whose subcellular localization was examined by immunofluorescence rather than GFP fluorescence. DIC, Nomarski differential interference contrast image; Chlorophyll, autofluorescence from phycobilin and chlorophyll; GFP, GFP fluorescence or immunofluorescence using anti-GFP antibody; Merge, merged images of autofluorescence and green fluorescence from the fusion protein. Bar $=2 \mu \mathrm{m}$. Abbreviation of enzyme names is listed in Table 1, Supplementary Table 3.

and CML329C were identified as KAS, condensing enzymes in the fatty acid synthesis. Analysis of subcellular localization indicated that CMM286C (KAS I) was targeted to the plastid, whereas CML329C (mtKAS) was localized in the mitochondrion (Supplementary Figure 2A). Additionally, C. merolae has a mitochondrial ACP (mtACP; CMS372C), which was targeted to the mitochondrion (Supplementary Figure 2A). These results show that $C$. merolae has fatty acid synthesis pathway in both the plastid and the mitochondrion (Figure 2), as in plants.

Because subcellular localization of stearoyl-CoA desaturase (SCD; CMM045C) was determined by GFP-fusion techniques with onion epidermal cells in a previous study (Sato and Moriyama, 2007), we confirmed it by analysis in C. merolae cells. There are two codons as candidates of initiator methionine in SCD. A polypeptide starting from the first methionine was localized in the cytosol, whereas a polypeptide starting from the second methionine was targeted to the ER (Supplementary Figure 2B), confirming that SCD is an ER-localized enzyme.

C. merolae has seven enzymes involved in fatty acid elongation (Table 1). KCS (CMD118C), 3-ketoacyl-CoA reductase (KCR; CMK172C), 3-hydroxyacyl-CoA dehydratase (HCD; CMR006C), and enoyl-CoA reductase (ECR; CMD146C) were localized in the ER (Supplementary Figure 2C). These results show that fatty acid elongation in C. merolae probably takes place in the ER (Figure 2), as in plants. A. thaliana has homologs of yeast ELO gene encoding KCS enzymes, but the function of most of them remains unclear (Quist et al., 2009; Haslam and Kunst, 2013). Three ELO-like homologs (CMT175C, CMM126C, and CML178C) were detected in the genomic data of C. merolae. Both CMT175C and CMM126C were targeted to the ER (Supplementary Figure 2C). We were not able to determine the subcellular localization of CML178C, because repeated attempts to detect fluorescence signals were unsuccessful. The $\mathrm{N}$-terminus of CML178C was longer than that of the other two homologs, and contained hydrophilic residues at its extremity. The failure of detection of GFP expression for the construct CML178C could be due 


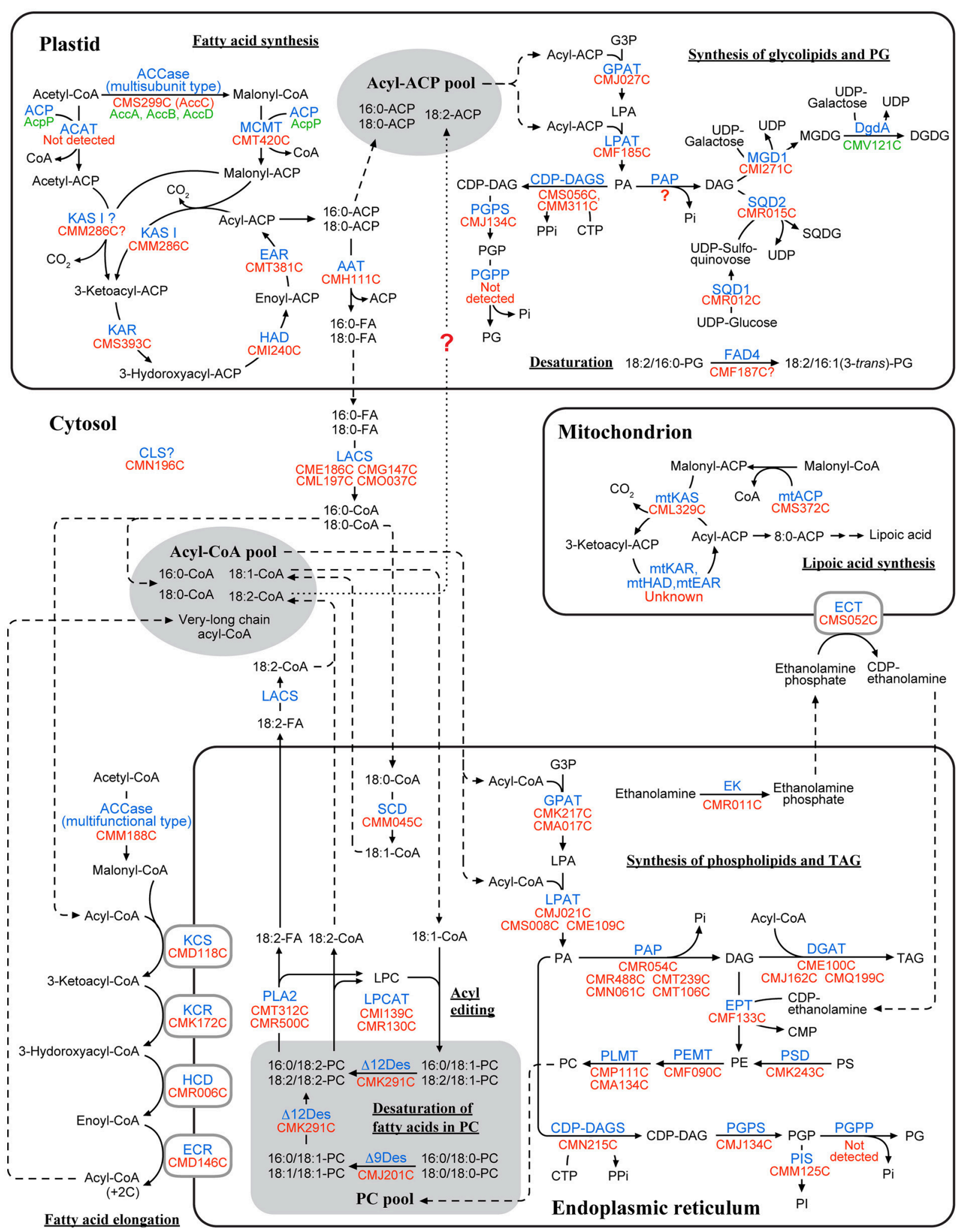

FIGURE 2 | A map of lipid metabolic pathways in C. merolae based on the results of subcellular localization analysis. This pathway map was created based on the results of subcellular localization analysis (Figure 1D, Supplementary Figures 2-6). Enzyme name is indicated in blue. Locus tag of the genes encoded in the nuclear and the plastid genomes are shown in red and green, respectively. Fatty acids are represented by a combination of the number of carbon atom [X] 


\section{FIGURE 2 | Continued}

and the number of double bonds [Y], such as X:Y. The number in the parenthesis indicates position of double bonds. Dashed line indicates flow of transport of substrates. In the plastidic fatty acid synthesis, it is possible that acetyl-CoA:ACP acetyltransferase (ACAT) converts acetyl-CoA to acetyl-ACP in the initial condensation reaction (see Discussion), although putative ACAT was not detected from the genomic data in C. merolae. Some enzymes related to mitochondrial fatty acid synthesis, mitochondrial enoyl-CoA reductase (mtEAR), mitochondrial 3-hydroxyacyl-ACP dehydratase (mtHAD), and mitochondrial 3-ketoacyl-ACP reductase (mtKAR), have not been identified either in plants or in C. merolae. In the synthesis of glycolipids and PG, phosphatidylglycerolphosphate phosphatase was not detected in the genomic data of C. merolae. No PAP candidates were localized in the plastid in C. merolae. Putative FAD4 enzyme is included in this figure, although its localization could not be experimentally determined. In C. merolae, linoleoyl-CoA is synthesized in the ER, and then is transferred to the plastid, but this transport system is largely unknown (dotted lines). Abbreviations: acyl-ACP, acyl-acyl carrier protein; acyl-CoA, acyl-coenzyme A; CDP-DAG, CDP-diacylglycerol; DAG, diacylglycerol; DGDG, digalactosyldiacylglycerol; FA, fatty acid; G3P, glycerol-3-phosphate; LPA, lysophosphatidic acid; LPL, lysophospholipid; MGDG, monogalactosyldiacylglycerol; PA, phosphatidic acid; PC, phosphatidylcholine; PE, phosphatidylethanolamine; PG, phosphatidylglycerol; PGP, phosphatidylglycerolphosphate; PI, phosphatidylinositol; PL, phospholipid; PS, phosphatidylserine; SQDG, sulfoquinovosyldiacylglycerol; TAG, triacylglycerol. Abbreviated names of enzyme and genes are shown according to Table 1, Supplementary Table 3.

to inefficient translation or accelerated degradation of the protein product.

\section{Synthesis of Glycolipids and PG}

In C. merolae, 10 enzymes involved in the synthesis of glycolipids and PG were identified by the Gclust analysis (Table 1). Most of these enzymes, namely lysophosphatidic acid acyltransferase (LPAT; CMF185C), UDP-sulfoquinovose synthase (SQD1; CMR012C), sulfoquinovosyldiacylglycerol synthase (SQD2; CMR015C) and CDP-diacylglycerol synthase (CDP-DAGS; CMS056C and CMM311C), were localized in the plastid (Supplementary Figure 2D). In plastidic CDPDAGSs, CMM311C was not analyzed, because the amino acid sequence of this protein is identical to that of CMS056C. Phosphatidylglycerolphosphate synthase (PGPS; CMJ134C) was dually localized in the plastid and cytosol (Supplementary Figure 2D). No PAP candidates were targeted to the plastid. DGDG synthase is encoded by a homolog of cyanobacterial $d g d A$ gene in the plastid genome (Sakurai et al., 2007), and was not analyzed. Glycerol-3-phosphate acyltransferase (GPAT; CMJ027C) was targeted to the plastid, as evidenced by the HA tag-fusion techniques (Supplementary Figure 2D). C. merolae has both enzymes of a plant type monogalactosyldiacylglycerol synthase (MGD1; CMI271C) and an MgdA homolog (CMT267C) that is one of the enzymes involved in MGDG synthesis in cyanobacteria. MGD1 was localized in the plastid, but the MgdA homolog was targeted to the ER (Supplementary Figure 2D). As the epimerase gene $M g d E$ was not detected in C. merolae, the function of $\mathrm{MgdA}$, if any, might not be related to the synthesis of MGDG (Sato and Awai, 2016). It is likely that MGDG synthesis is performed by MGD1 in $C$. merolae.

These results suggest that the plastid is the site of synthesis of glycolipids and PG in C. merolae (Figure 2), as in plants. No homolog of SFR2 gene encoding galactolipid:galactolipid galactosyltransfrase (Moellering et al., 2010) was detected in the genomic data of C. merolae. Recently, phosphatidylglycerolphosphate phosphatase has been identified in plants and green algae (Hung et al., 2015), but no homolog of this enzyme was detected in C. merolae. CMF187C was detected as a putative FAD4 protein catalyzing the synthesis of 16:1 (3-trans) in plastid PG (Gao et al., 2009). We detected this unsaturated fatty acid (Sato and Moriyama, 2007; Toyoshima et al., 2016), but, unfortunately, we were not able to detect the signal of CMF187C within the cell by repeated attempts.

\section{Synthesis of Phospholipids and TAG}

In C. merolae, 27 enzymes involved in the synthesis of phospholipids and TAG were detected by the Gclust analysis (Table 1). Most of these enzymes, for example GPAT (CMK217C and CMA017C), phosphatidylinositol synthase (PIS; CMM125C) and DGAT (CMQ199C, CME100C and CMJ162C), were targeted to the cytosol or ER (Figure 1D, Supplementary Figure 3). ECT was localized in the mitochondrion, as in plants (Mizoi et al., 2006; Figure 1D). No gene encoding phosphatidylserine synthase was detected in the genomic data of $C$. merolae, a fact which is consistent with the inability of detection of PS in $C$. merolae cells (Sato and Moriyama, 2007). In the same study, subcellular localizations of acyl-lipid $\Delta 9$ desaturase $(\Delta 9 \mathrm{Des}$; CMJ201C) and acyl-lipid $\Delta 12$ desaturase ( $\Delta 12$ Des; CMK291C) were analyzed by GFP-fusion techniques with onion epidermal cells (Sato and Moriyama, 2007), resulting in inconsistent localization depending on the selection of initiation codon. We now analyzed the subcellular localization of these desaturases in C. merolae cells. In $\Delta 9$ Des, both polypeptides starting from the first and the second methionines were localized in the ER (Supplementary Figure $2 B$ ). In $\Delta 12$ Des, both polypeptides starting from the first and the third methionines were localized in the ER, while a polypeptide starting from the second methionine was targeted to the cytosol (Supplementary Figure 2B). Based on these results, we consider that $\Delta 9$ Des and $\Delta 12$ Des are ER-localized enzymes. All these results suggest that the major site of synthesis of phospholipids and TAG must reside in the ER in C. merolae (Figure 2). This is a situation quite similar to that in plants, in contrast with mis-tuned targeting predictions (Table 1) and the results in heterologous expression systems.

In plants, $\mathrm{PC}$ is synthesized by both CDP-choline pathway and PE methylation pathway. However, C. merolae has no enzymes involved in the CDP-choline pathway, such as choline kinase (CK), phosphocholine cytidylyltransferase (CCT) and phosphoethanolamine methyltransferase (PEAMT). This suggests that PE methylation is the only identifiable pathway of PC synthesis in C. merolae (Figure 2), which was confirmed by labeling experiments using $\left[{ }^{32} \mathrm{P}\right]$ phosphate (Sato et al., 2016b). 
A

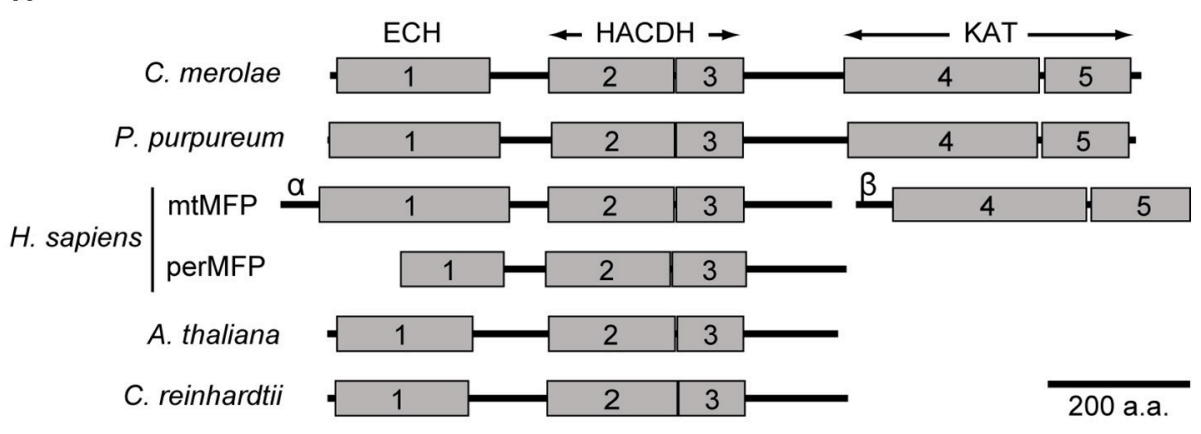

B

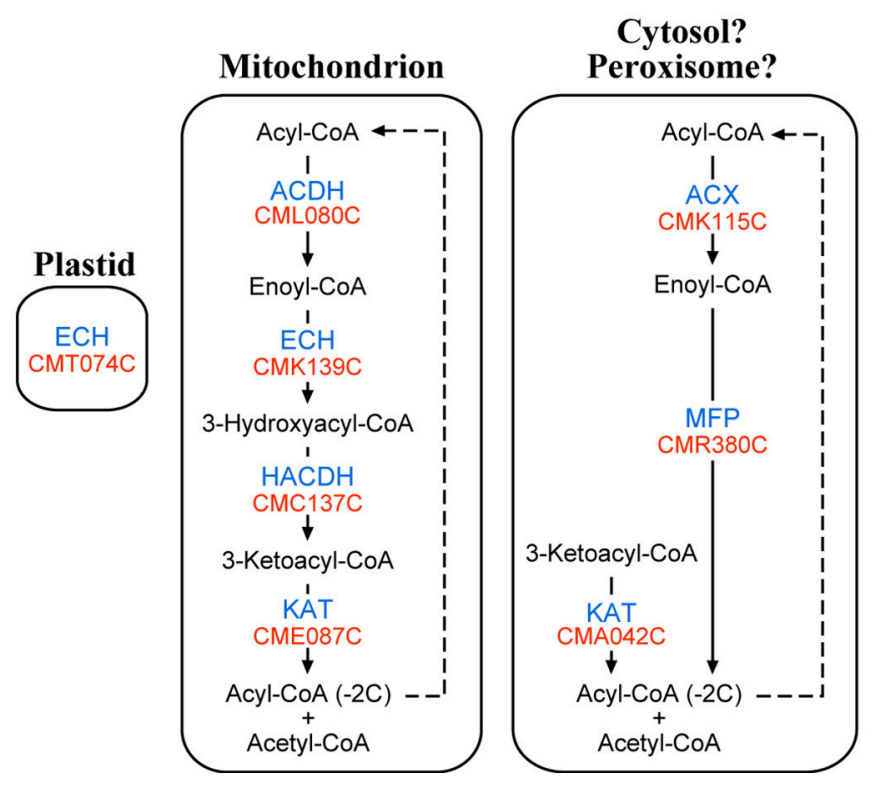

FIGURE 3 | $\boldsymbol{\beta}$-Oxidation of fatty acids in C. merolae. (A) Schematic domain structure of representative MFPs. We searched for functional domains of these MFPs using the Pfam database (Finn et al., 2014). Gray boxes indicate individual functional domains, namely enoyl-CoA hydratase/isomerase family domain (1),

3-hydroxyacyl-CoA dehydrogenase NAD binding domain (2), 3-hydroxyacyl-CoA dehydrogenase C-terminal domain (3), thiolase $N$-terminal domain (4), and thiolase $C$-terminal domain (5). Homo sapience has both peroxisomal MFP (perMFP) and mitochondrial MFP (mtMFP). mtMFP is a complex consisting of $\alpha$ and $\beta$ subunits ( $\alpha$ or $\beta$ ). (B) A pathway map of $\beta$-oxidation in C. merolae. This pathway map was created based on the results of subcellular localization analysis (Supplementary Figures 4A,B). For abbreviated names of enzymes and genes, see Table 1.

\section{$\beta$-Oxidation}

Nine enzymes related to $\beta$-oxidation of fatty acids were found in C. merolae by the Gclust analysis (Table 1). Four enzymes were localized in the mitochondrion, and CMC137C encoding 3hydroxyacyl-CoA dehydrogenase $(\mathrm{HACDH})$ was dually localized to the cytosol and mitochondrion (Supplementary Figure 4A). Other $\beta$-oxidation enzymes were targeted to the plastid or cytosol (Supplementary Figure 4A). Because it is possible that non-mitochondrial or non-plastid enzymes, namely acylCoA oxidase (ACX; CMK115C), multifunctional protein (MFP; CMR380C) and 3-ketoacyl-CoA thiolase (KAT; CMA042C), might be localized in the peroxisome, subcellular localization of these enzymes were analyzed with constructs of GFPfused $C$-terminal peptide (Supplementary Figure 4B). These enzymes, however, were not localized in the peroxisome in any constructs.

Figure 3A indicates schematic domain structure of MFPs in various organisms. Peroxisomal MFP in plants and animals has both enoyl-CoA hydratase $(\mathrm{ECH})$ and $\mathrm{HACDH}$ domains. In animals, mitochondrial MFP forms a heterooctamer complex consisting of $\alpha$ and $\beta$ subunits, of which the former subunit has $\mathrm{ECH}$ and HACDH domains, whereas a KAT domain exists in the latter subunit. By using the Pfam database (Finn et al., 2014), we found that C. merolae MFP has $\mathrm{ECH}, \mathrm{HACDH}$, and KAT domains within a single polypeptide chain. This suggests that $C$. merolae MFP might catalyze the three successive reactions of $\beta$ oxidation against saturated acyl-CoA as a substrate. The domain structure of $C$. reinhardtii MFP was similar to that of plant 
TABLE 2 | Poor performance of various prediction tools with respect to the localization of $C$. merolae proteins.

\begin{tabular}{|c|c|c|c|c|c|c|}
\hline \multirow{2}{*}{$\begin{array}{l}\text { Computer } \\
\text { program }\end{array}$} & \multirow[t]{2}{*}{ Prediction } & \multicolumn{4}{|c|}{ Confirmed subcellular localization } & \multirow{2}{*}{$\begin{array}{l}\text { Performance } \\
\text { score }\end{array}$} \\
\hline & & Pt & Mt & $\begin{array}{c}\text { ER and/or } \\
\text { CM }\end{array}$ & Cyt & \\
\hline \multirow[t]{4}{*}{ TargetP } & Pt (18) & 13 & 1 & 3 & 1 & 0.72 \\
\hline & Mt (38) & 10 & 11 & 15 & 2 & 0.29 \\
\hline & $\mathrm{SP}(7)$ & 0 & 0 & 5 & 2 & 0.71 \\
\hline & Other (43) & 4 & 2 & 22 & 15 & 0.35 \\
\hline \multirow{4}{*}{$\begin{array}{l}\text { WoLF } \\
\text { PSORT }\end{array}$} & Pt (44) & 16 & 8 & 14 & 6 & 0.36 \\
\hline & Mt (7) & 2 & 2 & 3 & 0 & 0.29 \\
\hline & ER, PM (27) & 4 & 0 & 16 & 7 & 0.59 \\
\hline & Cyt (12) & 3 & 1 & 3 & 5 & 0.42 \\
\hline \multirow[t]{4}{*}{ PredAlgo } & Pt (27) & 14 & 3 & 8 & 2 & 0.52 \\
\hline & Mt (17) & 4 & 4 & 6 & 3 & 0.24 \\
\hline & SP (16) & 1 & 2 & 13 & 0 & 0.81 \\
\hline & Other (46) & 8 & 5 & 18 & 15 & 0.33 \\
\hline
\end{tabular}

In the results of prediction using TargetP and PredAlgo, we considered that "SP" (secretory pathway) means targeting to the ER and/or the cytoplasmic membrane, and "Other" means targeting to the cytosol. In the results of WoLF PSORT, some enzymes predicted to localize in more than two compartments were not included in this rating. Abbreviations are listed in the legend for Table 1. Performance score is the proportion of correct localization.

homologs. Porphyridium purpureum, a red alga belonging to a different class from $C$. merolae contains an MFP, whose domain structure was similar to that in C. merolae MFP. It is likely that MFP having three domains in a single polypeptide chain is one of the features in red algae. Therefore, these results showed that C. merolae might have two $\beta$-oxidation pathways consisting of mitochondrial- and extra-mitochondrial-enzymes (Figure 3B).

\section{Other Enzymes Related to Acyl Lipid Metabolism}

Results of subcellular localization analysis of enzymes related to lipid degradation, lipid trafficking, fatty acid activation, PI signaling, and biotin-dependent carboxylation are reported in Supplementary Data 1, and summarized in Supplementary Table 3.

\section{DISCUSSION}

In the present study, we attempted to identify all the enzymes involved in acyl lipid metabolism in C. merolae and to analyze their subcellular localization as an initial basis for the future biochemical and genetic studies. We detected 121 enzymes involved in the metabolism of acyl lipids, and identified the subcellular localization of 110 of the enzymes by experimental analysis. These results suggest that the roles of different compartments in the synthesis of fatty acids and lipids are largely similar in C. merolae to those in plants, in spite of the strange predictions by various software, showing diversified localization. Table 2 indicates a comparison of the predictions of these computer programs with the results of subcellular localization analysis using the GFP- or HA tag-fusion techniques. Most of performance scores were less than 0.6. It seems likely that prediction of proteins localized in the plastid by TargetP was correct at a high rate (performance score; 0.72), but approximately half of the plastidic enzymes confirmed by experimental analysis were predicted as mitochondrial proteins by this program. The same is true of proteins in the secretory pathway. Although, they had a high performance score for secretory pathway proteins, TargetP and PredAlgo mispredicted proteins localized in the ER and/or the cytoplasmic membrane as target to mitochondrion and cytosol. These results suggest that these prediction tools for plants and green algae were not suitable for proteins in C. merolae, because they are not tuned or trained for the red alga. We consider that this is due to the fact that the targeting signals in this alga could be significantly different from those in both plants and green algae. In this respect, the results of the present study, as well as the results of a previous study (Moriyama et al., 2014a), will be useful as a training set for the neural network used in the prediction tools.

In this study, we constructed the acyl lipid metabolic map in C. merolae (Figure 2) based on comparative genomics and subcellular localization analysis, and found notable characteristics of lipid metabolism in this alga. However, homology-based identification has limitation in that they cannot theoretically predict detailed characteristics of the enzymes. Additional biochemical or genetic analyses will be required for elaborating this map. We discuss on the following topics: First, only a single enzyme KAS I being involved in the condensation reaction of fatty acid synthesis in the plastid. Second, pathways of TAG synthesis in C. merolae. Third, the sites of $\beta$-oxidation pathways in $C$. merolae. All these points will be important research projects for future biochemical and genetic studies.

\section{A Single KAS Enzyme Catalyzes Condensation Reactions in Plastidic Fatty Acid Synthesis}

We found KAS I and mtKAS, but not KAS II or KAS III, in C. merolae (Figure 2). Since condensation reactions of mitochondrial fatty acid synthesis are catalyzed solely by mtKAS in plants (Yasuno et al., 2004), it is expected that mitochondrial fatty acid synthesis in $C$. merolae might be catalyzed by a similar system. Plastidic fatty acid synthesis in plants involves three isoforms of KAS having different substrate specificities. Initial condensation reaction is catalyzed by KAS III, which catalyzes the formation of acetoacetyl-ACP from acetyl-CoA and malonyl-ACP (Clough et al., 1992). Subsequent condensations up to palmitoyl-ACP are catalyzed by KAS I, whereas the final elongation of palmitoyl-ACP to stearoyl-ACP requires KAS II (Shimakata and Stumpf, 1982; Pidkowich et al., 2007). Likewise, three isoforms of KAS participate in the fatty acid synthesis in Escherichia coli and cyanobacteria (Lem and Stumpf, 1984; Parsons and Rock, 2013). Because C. merolae lacks KAS II and KAS III, KAS I alone is supposed to catalyze all condensation reactions of fatty acid synthesis in the plastid. We tried to look for an alternative enzyme, acetyl-CoA:ACP acetyltransferase (ACAT), which could convert acetyl-CoA to acetyl-ACP in the 
initial condensation reaction, normally as part of the type I fatty acid synthase in animals and yeasts (Smith et al., 2003; Tehlivets et al., 2007), but we were not able to detect a homolog. As KAS I/II and KAS III are distinguished by functional domains in plants and bacteria, KAS III cannot be replaced by KAS I there. In Gram-negative pathogen Pseudomonas aeruginosa, FabY plays as an initial condensing enzyme in fatty acid synthesis, though it has a KAS I/II domain (Yuan et al., 2012). It is quite possible that $C$. merolae KAS I is able to catalyze the initial condensation reaction like FabY. Another question is related to the end product of fatty acid synthesis in C. merolae, if the final elongation from C16 to C18 is not catalyzed by KAS II. These points should be clarified by biochemical analysis using the plastids or isolated KAS I protein.

\section{Pathway of TAG Synthesis in C. merolae}

The simplest pathway of de novo TAG synthesis consists of four steps, the two acylations of glycerol-3-phosphate (G3P) by GPAT and LPAT, the dephosphorylation of phosphatidic acid by PAP, and the third acylation of DAG by DGAT or phospholipid:diacylglycerol acyltransferase (PDAT). PC is a key intermediate in the synthesis of TAG having polyunsaturated fatty acids, because PC is a major substrate of acyl lipid desaturase in ER (Bates et al., 2013). Transferring a polyunsaturated fatty acid from PC to DAG by PDAT is an important pathway of TAG synthesis via PC in plants. Phosphatidylcholine:diacylglycerol cholinephosphotransferase (PDCT) catalyzes transfer of a phosphocholine head group from PC to DAG, which is another important enzyme related to TAG synthesis via PC (Lu et al., 2009). This enzyme is able to produce DAG from unsaturated PC. PC-derived DAG are also produced by the degradation by phospholipase C (PLC) or phospholipase D (PLD) plus $\mathrm{PAP}$ and the reverse reaction of CPT. Genes encoding PDAT, PDCT, PLC, and PLD were not detected in C. merolae (Table 1, Supplementary Table 3). These results suggest that TAG is synthesized without the production of PC-derived DAG in $C$. merolae, although a large amount of TAG containing linoleic acid was detected in C. merolae by lipid analysis (Toyoshima et al., 2016). TAG could be produced by acylation of the DAG, which is

\section{REFERENCES}

Bates, P. D., Stymne, S., and Ohlrogge, J. (2013). Biochemical pathways in seed oil synthesis. Curr. Opin. Plant Biol. 16, 358-364. doi: 10.1016/j.pbi.2013. 02.015

Chapman, K. D., Dyer, J. M., and Mullen, R. T. (2012). Biogenesis and functions of lipid droplets in plants: thematic Review Series: Lipid Droplet Synthesis and Metabolism: from Yeast to Man. J. Lipid Res. 53, 215-226. doi: 10.1194/jlr.R021436

Clough, R. C., Matthis, A. L., Barnum, S. R., and Jaworski, J. G. (1992). Purification and characterization of 3-ketoacyl-acyl carrier protein synthase III from spinach. A condensing enzyme utilizing acetylcoenzyme A to initiate fatty acid synthesis. J. Biol. Chem. 267, 20992-20998.

Emanuelsson, O., Nielsen, H., Brunak, S., and von Heijne, G. (2000). Predicting subcellular localization of proteins based on their $\mathrm{N}$-terminal amino acid sequence. J. Mol. Biol. 300, 1005-1016. doi: 10.1006/jmbi. 2000.3903 formed from acyl CoA pool in equilibrium with the acyl groups in PC. This point will be another future project involving the metabolic flow of fatty acids in TAG synthesis in C. merolae.

\section{The Sites of $\beta$-Oxidation of Fatty Acids in C. merolae}

The results showed that $C$. merolae, unlike plants, might have two $\beta$-oxidation pathways consisting of mitochondrial- and extramitochondrial-enzymes (Figure 3B). The subcellular localization of the extra-mitochondrial $\beta$-oxidation enzymes, namely ACX, MFP, and KAT (CMA042C), were examined using the constructs of EGFP-fused $C$-terminal peptides of these enzymes, which were not localized in the peroxisome (Supplementary Figure $4 B$ ). No peroxisomal targeting signals (Lingner et al., 2011) were detected from $\beta$-oxidation enzymes except for ACX in C. merolae. Additionally, these enzymes were not detected by proteome analysis of isolated C. merolae peroxisomes (Imoto et al., 2013). These might suggest that the sites of extra-mitochondrial $\beta$ oxidation pathways might be in the cytosol in C. merolae, as in bacteria. This should be investigated experimentally, because this is an unprecedented situation.

\section{AUTHOR CONTRIBUTIONS}

All authors listed, have made substantial, direct and intellectual contribution to the work, and approved it for publication.

\section{ACKNOWLEDGMENTS}

This work was supported by in part by the Core Research for Evolutional Science and Technology (CREST) from the Japan Science and Technology Agency (JST).

\section{SUPPLEMENTARY MATERIAL}

The Supplementary Material for this article can be found online at: http://journal.frontiersin.org/article/10.3389/fpls.2016. 00958

Finn, R. D., Bateman, A., Clements, J., Coggill, P., Eberhardt, R. Y., Eddy, S. R., et al. (2014). Pfam: the protein families database. Nucleic Acids Res. 42, 222-230. doi: 10.1093/nar/gkt1223

Gao, J., Ajjawi, I., Manoli, A., Sawin, A., Xu, C., Froehlich, J. E., et al. (2009). FATTY ACID DESATURASE4 of Arabidopsis encodes a protein distinct from characterized fatty acid desaturases. Plant J. 60, 832-839. doi: 10.1111/j.1365313X.2009.04001.x

Graham, I. A., and Eastmond, P. J. (2002). Pathways of straight and branched chain fatty acid catabolism in higher plants. Prog. Lipid Res. 41, 156-181. doi: 10.1016/S0163-7827(01)00022-4

Gueguen, V., Macherel, D., Jaquinod, M., Douce, R., and Bourguignon, J. (2000). Fatty acid and lipoic acid biosynthesis in higher plant mitochondria. J. Biol. Chem. 275, 5016-5025. doi: 10.1074/jbc.275.7.5016

Haslam, T. M., and Kunst, L. (2013). Extending the story of very-long-chain fatty acid elongation. Plant Sci. 210, 93-107. doi: 10.1016/j.plantsci.2013.05.008

Horton, P., Park, K. J., Obayashi, T., Fujita, N., Harada, H., Adams-Collier, C. J., et al. (2007). WoLF PSORT: Protein localization predictor. Nucleic Acids Res. 35, W585-W587. doi: 10.1093/nar/gkm259 
Hung, C.-H., Kobayashi, K., Wada, H., and Nakamura, Y. (2015). Isolation and characterization of a phosphatidylglycerophosphate phosphatase1, PGPP1, in Chlamydomonas reinhardtii. Plant Physiol. Biochem. 92, 56-61. doi: 10.1016/j.plaphy.2015.04.002

Imoto, Y., Kuroiwa, H., Yoshida, Y., Ohnuma, M., Fujiwara, T., Yoshida, M., et al. (2013). Single-membrane-bounded peroxisome division revealed by isolation of dynamin-based machinery. Proc. Natl. Acad. Sci. U.S.A. 110, 9583-9588. doi: $10.1073 /$ pnas. 1303483110

Itoh, R., Toda, K., Takahashi, H., Takano, H., and Kuroiwa, T. (1998). Delta-9 fatty acid desaturase gene containing a carboxyl-terminal cytochrome b5 domain from the red alga Cyanidioschyzon merolae. Curr. Genet. 33, 165-170. doi: $10.1007 / \mathrm{s} 002940050323$

Joyard, J., Ferro, M., Masselon, C., Seigneurin-Berny, D., Salvi, D., Garin, J., et al. (2010). Chloroplast proteomics highlights the subcellular compartmentation of lipid metabolism. Prog. Lipid Res. 49, 128-158. doi: 10.1016/j.plipres.2009.10.003

Katayama, K., Sakurai, I., and Wada, H. (2004). Identification of an Arabidopsis thaliana gene for cardiolipin synthase located in mitochondria. FEBS Lett. 577, 193-198. doi: 10.1016/j.febslet.2004.10.009

Larkin, M. A., Blackshields, G., Brown, N. P., Chenna, R., Mcgettigan, P. A., McWilliam, H., et al. (2007). Clustal W and Clustal X version 2.0. Bioinformatics 23, 2947-2948. doi: 10.1093/bioinformatics/btm404

Lem, N. W., and Stumpf, P. K. (1984). In vitro fatty acid synthesis and complex lipid metabolism in the cyanobacterium Anabaena variabilis: I. Some characteristics of fatty acid synthesis. Plant Physiol. 74, 134-138. doi: 10.1104/pp.74.1.134

Li-Beisson, Y., Beisson, F., and Riekhof, W. (2015). Metabolism of acyl-lipids in Chlamydomonas reinhardtii. Plant J. 84, 504-522. doi: 10.1111/tpj.12787

Li-Beisson, Y., Shorrosh, B., Beisson, F., Andersson, M. X., Arondel, V., Bates, P. D., et al. (2013). Acyl-Lipid Metabolism. Arab. B. 11:e0161. doi: 10.1199/tab.0161

Lingner, T., Kataya, A. R., Antonicelli, G. E., Benichou, A., Nilssen, K., Chen, X.-Y., et al. (2011). Identification of novel plant peroxisomal targeting signals by a combination of machine learning methods and in vivo subcellular targeting analyses. Plant Cell 23, 1556-1572. doi: 10.1105/tpc.111. 084095

Lu, C., Xin, Z., Ren, Z., Miquel, M., and Browse, J. (2009). An enzyme regulating triacylglycerol composition is encoded by the ROD1 gene of Arabidopsis. Proc. Natl. Acad. Sci. U.S.A. 106, 18837-18842. doi: 10.1073/pnas.0908848106

Matsuzaki, M., Misumi, O., Shin,-I., T., Maruyama, S., Takahara, M., Miyagishima, S.-Y., et al. (2004). Genome sequence of the ultrasmall unicellular red alga Cyanidioschyzon merolae 10D. Nature 428, 653-657. doi: 10.1038/nature02398

Mizoi, J., Nakamura, M., and Nishida, I. (2006). Defects in CTP:PHOSPHORYLETHANOLAMINE CYTIDYLYLTRANSFERASE affect embryonic and postembryonic development in Arabidopsis. Plant Cell 18, 3370-3385. doi: 10.1105/tpc.106.040840

Moellering, E. R., Muthan, B., and Benning, C. (2010). Freezing tolerance in plants requires lipid remodeling at the outer chloroplast membrane. Science 330, 226-228. doi: 10.1126/science. 1191803

Moriyama, T., Sakurai, K., Sekine, K., and Sato, N. (2014a). Subcellular distribution of central carbohydrate metabolism pathways in the red alga Cyanidioschyzon merolae. Planta 240, 585-598. doi: 10.1007/s00425-014-2108-0

Moriyama, T., Tajima, N., Sekine, K., and Sato, N. (2014b). Localization and phylogenetic analysis of enzymes related to organellar genome replication in the unicellular rhodophyte Cyanidioschyzon merolae. Genome Biol. Evol. 6, 228-237. doi: 10.1093/gbe/evu009

Nakamura, Y., Sasaki, N., Kobayashi, M., Ojima, N., Yasuike, M., Shigenobu, Y., et al. (2013). The first symbiont-free genome sequence of marine red alga, Susabi-nori (Pyropia yezoensis). PLoS ONE 8:e57122. doi: 10.1371/journal.pone.0057122

Nozaki, H., Takano, H., Misumi, O., Terasawa, K., Matsuzaki, M., Maruyama, S., et al. (2007). A 100\%-complete sequence reveals unusually simple genomic features in the hot-spring red alga Cyanidioschyzon merolae. BMC Biol. 5:28. doi: 10.1186/1741-7007-5-28

Ohnuma, M., Yokoyama, T., Inouye, T., Sekine, Y., Kuroiwa, T., and Tanaka, K. (2014). Optimization of polyethylene glycol (PEG)-mediated DNA introduction conditions for transient gene expression in the unicellular red alga Cyanidioschyzon merolae. J. Gen. Appl. Microbiol. 60, 156-159. doi: 10.2323/jgam.60.156

Ohnuma, M., Yokoyama, T., Inouye, T., Sekine, Y., and Tanaka, K. (2008). Polyethylene glycol (PEG)-mediated transient gene expression in a red alga, Cyanidioschyzon merolae 10D. Plant Cell Physiol. 49, 117-120. doi: $10.1093 / \mathrm{pcp} / \mathrm{pcm} 157$

Parsons, J. B., and Rock, C. O. (2013). Bacterial lipids: metabolism and membrane homeostasis. Prog. Lipid Res. 52, 249-276. doi: 10.1016/j.plipres.2013. 02.002

Pidkowich, M. S., Nguyen, H. T., Heilmann, I., Ischebeck, T., and Shanklin, J. (2007). Modulating seed $\beta$-ketoacyl-acyl carrier protein synthase II level converts the composition of a temperate seed oil to that of a palm-like tropical oil. Proc. Natl. Acad. Sci. U.S.A. 104, 4742-4747. doi: 10.1073/pnas.061 1141104

Poirier, Y., Antonenkov, V. D., Glumoff, T., and Hiltunen, J. K. (2006). Peroxisomal $\beta$-oxidation-A metabolic pathway with multiple functions. Biochim. Biophys. Acta Mol. Cell Res. 1763, 1413-1426. doi: 10.1016/j.bbamcr.2006.08.034

Quist, T. M., Sokolchik, I., Shi, H., Joly, R. J., Bressan, R. A., Maggio, A., et al. (2009). HOS3, an ELO-like gene, Inhibits effects of ABA and implicates a S-1$\mathrm{P} /$ ceramide control system for abiotic stress responses in Arabidopsis thaliana. Mol. Plant 2, 138-151. doi: 10.1093/mp/ssn085

Sakurai, I., Mizusawa, N., Wada, H., and Sato, N. (2007). Digalactosyldiacylglycerol is required for stabilization of the oxygen-evolving complex in photosystem II. Plant Physiol. 145, 1361-1370. doi: 10.1104/pp.107.106781

Sato, N. (2009). Gclust: trans-kingdom classification of proteins using automatic individual threshold setting. Bioinformatics 25, 599-605. doi: 10.1093/bioinformatics/btp047

Sato, N., and Awai, K. (2016). Diversity in biosynthetic pathways of Galactolipids in the Light of Endosymbiotic Origin of Chloroplasts. Front. Plant Sci. 7:117. doi: $10.3389 /$ fpls.2016.00117

Sato, N., Kobayashi, S., Aoki, M., Umemura, T., Kobayashi, I., and Tsuzuki, M. (2016a). Identification of genes for sulfolipid synthesis in primitive red alga Cyanidioschyzon merolae. Biochem. Biophys. Res. Commun. 470, 1-7. doi: 10.1016/j.bbrc.2016.01.006

Sato, N., Mori, N., Hirashima, T., and Moriyama, T. (2016b). Diverse pathways of biosynthesis of phosphatidylcholine in algae as estimated by labeling studies and genomic sequence analysis. Plant J. doi: 10.1111/tpj.13199. [Epub ahead of print].

Sato, N., and Moriyama, T. (2007). Genomic and biochemical analysis of lipid biosynthesis in the unicellular rhodophyte Cyanidioschyzon merolae: Lack of a plastidic desaturation pathway results in the coupled pathway of galactolipid synthesis. Eukaryot. Cell 6, 1006-1017. doi: 10.1128/EC.00393-06

Shimakata, T., and Stumpf, P. K. (1982). Isolation and function of spinach leaf $\beta$-ketoacyl-[acyl-carrier-protein] synthases. Proc. Natl. Acad. Sci. U.S.A. 79, 5808-5812. doi: 10.1073/pnas.79.19.5808

Smith, S., Witkowski, A., and Joshi, A. K. (2003). Structural and functional organization of the animal fatty acid synthase. Prog. Lipid Res. 42, 289-317. doi: 10.1016/S0163-7827(02)00067-X

Tardif, M., Atteia, A., Specht, M., Cogne, G., Rolland, N., Brugiere, S., et al. (2012). PredAlgo: a new subcellular localization prediction tool dedicated to green algae. Mol. Biol. Evol. 29, 3625-3639. doi: 10.1093/molbev/mss178

Tehlivets, O., Scheuringer, K., and Kohlwein, S. D. (2007). Fatty acid synthesis and elongation in yeast. Biochim. Biophys. Acta Mol. Cell Biol. Lipids 1771, 255-270. doi: 10.1016/j.bbalip.2006.07.004

Toyoshima, M., Mori, N., Moriyama, T., Misumi, O., and Sato, N. (2016). Analysis of TAG accumulation under nitrogen deprivation in the red alga Cyanidioschyzon merolae. Microbiology. 162, 803-812. doi: $10.1099 /$ mic. 0.000261

Wada, H., Shintani, D., and Ohlrogge, J. (1997). Why do mitochondria synthesize fatty acids? Evidence for involvement in lipoic acid production. Proc. Natl. Acad. Sci. U.S.A. 94, 1591-1596. doi: 10.1073/pnas. 94.4.1591

Watanabe, S., Ohnuma, M., Sato, J., Yoshikawa, H., and Tanaka, K. (2011). Utility of a GFP reporter system in the red alga Cyanidioschyzon merolae. J. Gen. Appl. Microbiol. 57, 69-72. doi: 10.2323/jgam.57.69

Yagisawa, F., Fujiwara, T., Kuroiwa, H., Nishida, K., Imoto, Y., and Kuroiwa, T. (2012). Mitotic inheritance of endoplasmic reticulum in the primitive red alga 
Cyanidioschyzon merolae. Protoplasma 249, 1129-1135. doi: 10.1007/s00709011-0359-1

Yasuno, R., Von Wettstein-Knowles, P., and Wada, H. (2004). Identification and molecular characterization of the $\beta$-ketoacyl-[acyl carrier protein] synthase component of the Arabidopsis mitochondrial fatty acid synthase. J. Biol. Chem. 279, 8242-8251. doi: 10.1074/jbc.M308894200

Yuan, Y., Sachdeva, M., Leeds, J. A., and Meredith, T. C. (2012). Fatty acid biosynthesis in Pseudomonas aeruginosa is initiated by the faby class of $\beta$ ketoacyl acyl carrier protein synthases. J. Bacteriol. 194, 5171-5184. doi: 10.1128/JB.00792-12
Conflict of Interest Statement: The authors declare that the research was conducted in the absence of any commercial or financial relationships that could be construed as a potential conflict of interest.

Copyright (c) 2016 Mori, Moriyama, Toyoshima and Sato. This is an open-access article distributed under the terms of the Creative Commons Attribution License (CC $B Y)$. The use, distribution or reproduction in other forums is permitted, provided the original author(s) or licensor are credited and that the original publication in this journal is cited, in accordance with accepted academic practice. No use, distribution or reproduction is permitted which does not comply with these terms. 